\title{
Editorial
}

\section{THE FUTURE OF ANATOMY}

\author{
Gustavo H. R. A. Otegui
}

University of Buenos Aires, Buenos Aires, Argentina

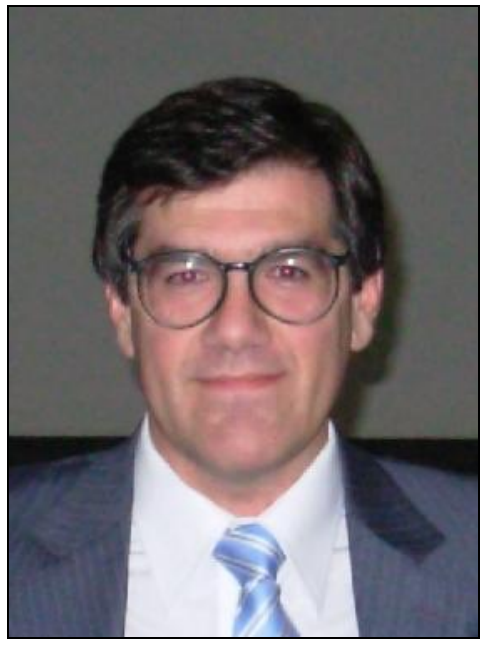

What could be expected from anatomy within the next few decades? We'll discourse an answer to what possible paths may the future of anatomy take, what scientific problems we'll have to take on, how should we learn and teach anatomy and what possible applications may come from our daily efforts.

Our approach was done within these three dimensions.

\section{Anatomy as a science}

Anatomy primarily study shape, order and the structure within a system.

Its research method is dissection; but as Eduardo D. P. De Robertis once said, "Those who work in the field of macroscopic, microscopic or molecular anatomy, tend to isolate their subject of interest. The method is the same whether one uses a scalpel to dissect a cadaver, or slices a sample in slides to see through an optic or electron microscope, or whether the separation is done by homogenization or centrifugation of subcellular components" (De Robertis, 1981). H.S.
Bennett said, "the operational methods from every branch of anatomy have similar characteristics" (De Robertis, 1981). Anatomy is a way of thinking; it's a research style, a way of explaining and understanding reality. As Francis Crick (who along with James Watson were awarded in 1962 with the Nobel Prize in Medicine for the development of a model for a helical structure of DNA) once said, "If you want to understand the function, study the structure." (Crick, 1989). On that note, another preeminent scientist, Albert Szent Györgi, a 1937 Nobel Laureate who was awarded for his discoveries in connection with the biological combustion processes, with special reference to vitamin C and the catalysis of fumaric acid, used to say "If the structure does not tell you something about the function, it means that you haven't looked hard enough", (Geneser, 2000).

Those who argue that the anatomy must inevitably be studied by memory, have done much harm to our discipline. Long lists of terminology applied to identify structures are demanded to be memorized, as well as charts with myriad of muscle insertions, vascular irrigations, innervations, relations, etc. This would be the same as to demand from every chemistry graduate to remember the atomic weight and properties of each of the existing atom in the table. To perpetuate the metaphor that the anatomy acts as a G.P.S. for the surgical practice is diminishing up to the point of turning anatomy into a map or data base for surgeons. But anatomy is much more than that. It's a science. It hopes to answer those relevant questions that could help us to understand and explain why are we the way we are. Anatomy's method comes from dissecting (from Latin, dis: to separate and section: part) whether or not it's done using a scalpel; meaning that its methodology leans on 
reductionism, it takes an analytical approach. But after any division of parts is done, the anatomists have to rebuild the whole, because they know that that's where system properties may emerge. This practice could be classified ontologically as emergentist. We disassemble in order to understand the laws that rule over a system build; to comprehend how its parts interact, basically how it works. By conducting himself in this manner was William Harvey able to postulate his theory on the circulatory system which is found in the sublime "Exercitatio Anatomica de Motu Cordis et Sanguinis in Animalibus" (Harvey, 1628); a must-read among anatomists, who should also recommend it to their students for its logical constructs, its coherence and the beauty of the anatomic demonstrations shown.

Following this procedures, Wernicke postulated his first model for the anatomy of language. This model was constituted by three components: Broca's area (expressive area), Wernicke's area (receptive area) and the arcuate fasciculus connecting the former with the latter. Also, neither an electrocardiogram nor its waves can be understood if one does not know the anatomy involved in the cardiac conduction system.

To sum up, we have established that as anatomists,our discipline involves a reductionist methodology as well as an emergentist ontology. Each organizational level within the subject possesses a structure that allows you to understand a key function of said level. An arm's abduction can be understood by analysing the disposition that the deltoid and the supraspinatus muscles take but muscle contraction is comprehended once we grasp the concept of the structure of a sarcomere. Language is comprehended once we have a basic understanding of the anatomic model proposed by Wernicke but inter-neural communication and the transmission of a nervous impulse can only be comprehended when we have studied the synapse on an ultra-structural level. The Human Anatomy Syllabi of the future shall take a progressive stance and they'll include other disciplines such as Genetics, Immunology, Physiology, Pharmacology, etc. To illustrate our point, there are some scientific problems that we have already began to deal with: the Human Connectome Project is trying to shed a light over the connectivity of every nucleus and brain area; in the field of biochemical anatomy, the distribution on the human body of some receptor or molecular signal on a macroscopic scale or the formula that determines the macroscopic map for the differential and harmonic expression of any given gene. Also the program that's trying to explain the macroscopic arrangement of the bundle of myocardium fibers, etc
This research programs will allow us to answer new questions and they will expand the horizons of our anatomic knowledge.

Imaging techniques such as spectroscopy, 7 or 9 Tesla MRI (still in experimental stages) will allow us a live view into the microscopic anatomy of a patient. In the future, we shall increase our ability to analyze functional anatomy on live subjects.

We will be able to rationally understand why certain drugs act, or not, on any given organ or why do some adverse effects could develop.

\section{To learn and teach anatomy}

Little is known about the process involved in learning anatomy and therefore how to teach it better. We have barely taken our first steps towards didactic morphology. In the field of didactic resources, new applied learning technologies such as virtual dissecting tables like anatomage $^{\mathrm{TM}}$, augmented reality lenses like hololens $^{T M}$ (Microsoft corp.) or biosimulators shall innovate in ways we cannot imagine yet. The ubiquity of learning and teaching will let us subdivide their relationship like we never have before. Biosimulation and virtual reality will enable students to make their own virtual gross dissections anywhere and anytime they please. These gross dissections shall be as realistic as are the ones done nowadays on cadavers. 3D printing is revolutionizing anatomy learning by creating and recreating life-like renderings. This renderings are so accurate that some are used as custom made prosthesis for people who have lost a limb. We shall develop personalized education in order to stimulate our students' creativity and different styles of learning.

Applications, innovations and developments for anatomy (medical consult and technoanatomy)

From the integration with cellular and molecular biology, genetics and developmental biology, tissue biotechnology and bioengineering new prosthesis and organs will be developed. Anatomy will allow the creation of new organ, tissue and stem-cell banks for research and development of prosthetics. New materials will be develop that will work as tisularstromas to organize the cells to shape the organs needed. In addition, anatomy will assist to diagnostics, therapeutic and minimally invasive robotically mediated surgery.

Those who work on image diagnostics will need to improve their ability to interpret the new techniques that will come along with these new technologies.

Anatomy oriented toward sports, nutrition, motor or postural rehabilitation, phoniatrics, among other will be developed enormously.

Therefore, contrary to belief, an anatomy well integrated with other disciplines is continuously 
and increasingly pushing the boundaries of what is known and the field of work for those in it.

\section{Bibliography}

Crick F. 1989. Qué loco propósito. Una visión personal del descubrimiento científico. Barcelona. España. Tusquets editores. 1a Ed. 171.
De Robertis EDP; De Robertis EMF. 1981. Biología Celular. Buenos Aires. Argentina. Editorial El Ateneo. 2 $2^{\mathrm{a}}$ Ed. 5.

Geneser F.2000. Histología. Buenos Aires. Argentina. Editorial Médica Panamericana. 1ㄹ Ed. 51.

Harvey .1628. Motu Cordis. Estudio anatómico del movimiento del corazón y de la sangre en los animales. Buenos Aires. Argentina. Editorial Universitaria de Buenos Aires. Reimpresión 1970. 